\title{
A rare case of squamous cell carcinoma of the paratesticular tissues
}

\section{Introduction}

Tumors of the testis and paratesticular tissues are rare neoplasms. Malignant testicular tumors (MTT) comprise approximately $1 \%-2 \%$ of all human neoplasms and $5 \%$ of the tumors of the urinary system and male reproductive organs. Most MTTs develop at 20-30 years of age. MTTs are the leading cause of death from cancer in individuals aged 14-44 years. The potential risk factors for the development of MTTs include family history, previous medical history of the tumor in the contralateral testicle, and components of the testicular dysgenesis syndrome (cryptorchidism, hypospadias, and spermatogenesis disorders, leading to infertility). Tumors of the paratesticular tissues are considerably less common and in most cases, are benign.

Squamous cell carcinoma rarely occurs as a primary tumor of the testicle and/or paratesticular tissues; few cases have been reported thus far. More commonly, there is a tumor secondary to the scrotal organs; however, these clinical situations are commonly observed in elderly patients.

\section{Clinical case}

Patient G., who was 27 years old, had no previous medical history, and had swelling in the left testicle for the previous 6 months visited our center. He had not undergone any treatment previously. During the previous 1.5 months, the tumor in his left testicle had started to increase in size, and he was admitted urgently to the neurology department with pain in the left lumbar region. Fine-needle multifocal biopsy of the left testis was performed, and the pathological reports showed aplastic cells of a malignant tumor, while cytological examination demonstrated embryonic cancer.

He was admitted to the urology unit of an oncological institution for further diagnostic procedures and treatment. Computer tomography showed lesions in S2 and S6 of the right lung as well as in S1-2 and S10 of the left lung. Few foci up to $4 \mathrm{~mm}$ and enlarged paraortal

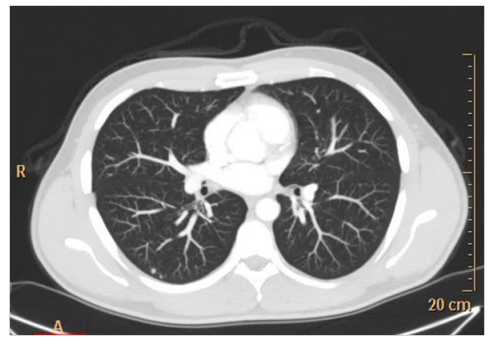

Figure I Nodules in the lung parenchyma.

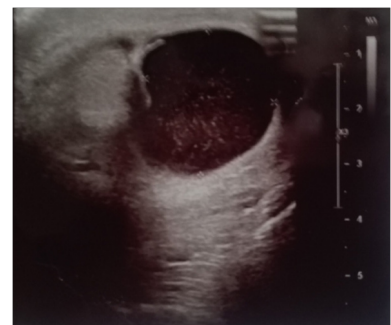

Figure 3 Tumor of the testis. Ultrasonogrpahic visualization.
Volume 9 Issue 2 - 2018

\section{Artemyeva AS, Mamizhev EM, Nosov A K, Khokhlova AV, Kushnarev VA, Kharchenko EV, Protsenko SA, Semiglazova TYu \\ Federal State Budget Institution, National Medical Research Center of Oncology na N.N. Petrov, Ministry of Healthcare of Russian Federation, Russia}

Correspondence: Semiglazova Tatiana Yurievna, PhD, ScD, MD, Head of Department, Leading Researcher, Department of Innovative Methods of Therapeutic Oncology and Rehabilitation, Federal State Budget Institution "National Medical Research Center of Oncology na N.N. Petrov," Ministry of Healthcare of Russian Federation, Email tsemiglazova@mail.ru

Received: February 24, 2018| Published: March 23, 2018

lymph nodes up to $27 \times 23 \mathrm{~mm}$ were observed (Figure 1) (Figure 2). According to the ultrasonographic examination of the scrotum: the right testicle and epididymis had no pathology; the left testicle was $57 \times 41 \mathrm{~mm}$ in size. There was isoechoic formation $(48 \times 37 \mathrm{~mm})$, heterogeneous structure due to the hypoechoic areas with fuzzy contours. Under the tunics, a moderate amount of liquid with a finedispersed suspension was determined; in the projection of the tail of the epididymis, there was an echogenic formation $(26 \times 23 \mathrm{~mm})$ with heterogeneous fine-dispersed contents (Figure 3). The patient's fertility status was assessed: blood tests showed that the levels of luteinizing hormone, follicle stimulating hormone, testosterone, and oncomarkers (alpha-fetoprotein [AFP], lactate dehydrogenase $[\mathrm{LDH}]$, and $\beta$ human chorionic gonadotropin $[\beta-\mathrm{hCG}])$ were normal. Spermography showed normospermia.

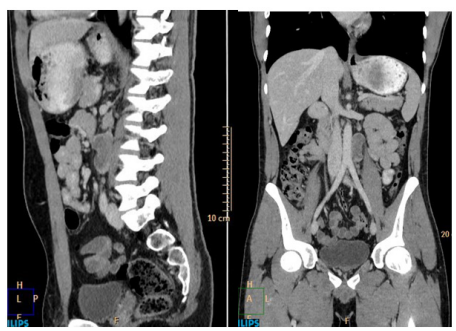

Figure 2 Enlarged retroperitoneal lymph nodes.

Radical inguinal orchiectomy was performed. The pathology report included macroscopic and microscopic assessment. The left testicle was $10 \times 60 \times 40 \mathrm{~mm}$, and the tunica vaginalis was damaged in the projection of the tumor. The tumor size was $65 \times 40 \times 50 \mathrm{~mm}$ with necrosis and hemorrhages, gray-yellow with the invasion of the tunica vaginalis of the testicle. Epididymis was not determined, replaced by a tumor. In the projection of the epididymis, the tumor sprouted into the tunica vaginalis. Microscopic assessment: the tumor was represented by a high-grade carcinoma, predominantly of a solid structure with portions of the biphasic structure owing to the presence of the sarcomatoid component. The tumor had extensive 
areas of necrosis and fibrosis zones. The tumor was localized in the paratesticular region, with the invasion of the testicular parenchyma, tunica vaginalis, and albuginea. The residual tissue of the epididymis was identified as having fibrosis, epithelial atrophy, and tumor infiltration. The spermatic cord was intact; further, the margin of resection of the spermatic cord was also intact. We did not observe any lymphovascular invasion. Teratoma structures were not found.

Immunohistochemical examination of MCK, vimentin, p53, EMA

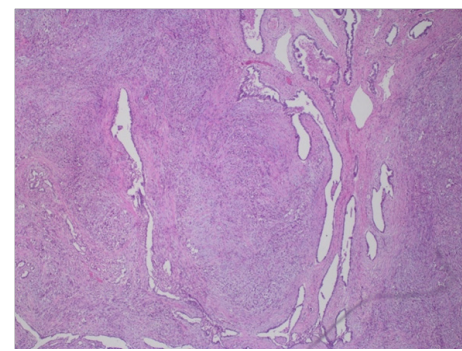

Figure 4 Tumor infiltrating the rete testis. H\&E $\times 200$.

In order to exclude the primary tumor outside the scrotum, diagnostic examinations, including bronchoscopy, gastroscopy, colonoscopy, and cystoscopy were performed. There were no indications of primary tumor outside the scrotum. PCRHPV blood test was negative. Уженаписалиобэтом Considering all the clinical and diagnostic data, a final diagnosis was established as follows: "Lowgrade non-keratinized squamous cell carcinoma of the left testicle with metastasis to the retroperitoneal lymph nodes and the lungs."

The patient was treated with PF (cisplatin $50 \mathrm{mg}+5$-fluorouracili $1800 \mathrm{mg}$ ). At the time of writing this report, the first cycle of chemotherapy had been administered. The effect of chemotherapy will be assessed after 3 cycles.

\section{Discussion}

Squamous cell carcinoma of the scrotum organs is extremely rare. In most cases, this occurs as a metastasis from the primary cell tumors of the lung, pelvis, or genitals, usually observed in elderly patients. ${ }^{2,3}$ In young patients, the most frequent tumors for this localization are germinal cell tumors. It is necessary to exclude teratoma with a somatic malignancy. This variant of malignant transformation develops relatively rarely and is observed in approximately $3 \%-6 \%$ of germinal cell testicular tumors. More commonly, the malignant transformation is accompanied by progression with systemic treatment; however, it may also occur primarily in teratoma. Most often, the malignant component is represented by sarcomas, and in very rare cases, it is represented by adenocarcinoma and squamous cell carcinoma. ${ }^{4,5}$ Tumors of the paratesticular tissue are much less common than the testicular tumors, and most of them are benign. The most frequent tumor of the paratesticular zone is an adenomatoid tumor that has a mesothelial origin. Epithelial tumors of the epididymis are cystadenoma, papillary cystadenoma, and adenocarcinoma. There is one well-documented case of squamous cell carcinoma of the epididymis in an 86-year-old man with a background of chronic hydrocele. ${ }^{6}$

This case can be considered interesting because the background processes were not revealed. At the time of diagnosis, the tumor was quite large; however, there were no data regarding the location of the primary tumor. Clinically, this case can be regarded as a situation of "diagnostic delay," delayed referral to an oncologist and improper diagnostic testicular biopsy examination in a non-specialized (foci), CK HMW, and p40 gave positive results; Sall4, OCT3/4, D240, CD30, GCP3, CD117, calretinin, S-100, WT-1, PAX-8, CDx2, CK7, and p16 were negative; Ki67 was 90\%. P53 was 90\%.

The final pathology examination showed poorly differentiated non-keratinized squamous cell carcinoma of the paratesticular tissues, probably an epididymis, with parenchyma of the testis and tunica vaginalis invasion. ICD-O code 8070/3 (Figure 4) (Figure 5).

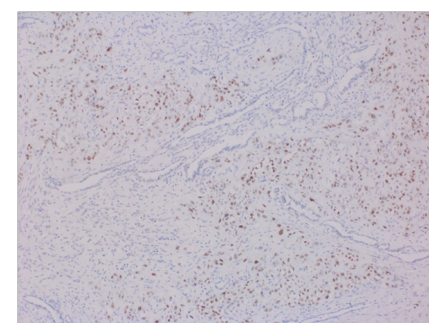

Figure 5 Tumor infiltrating the rete testis. IHC p40 staining $\times 200$.

institution. Timely and correctly performed therapy, including surgery with chemotherapy can improve the outcomes considerably? Moreover, in some cases, patients are embarrassed to visit the doctor, and this can lead to delay in treatment.

The prognosis of patients with metastatic squamous cell carcinoma is worse than that of patients with germ cell tumors at a similar stage. Improving the diagnostic consideration and introduction of new therapeutic agents can potentially improve the situation.

\section{Acknowledgements}

None.

\section{Conflict of interest}

Authors have declared no conflicts of interest.

\section{References}

1. WHO classification of tumours of the urinary system and male genital organs. In: Holger Moch, Peter A Humphrey, Thomas M Ulbright, et al. editors. IARC. Lyon, 2016.

2. Ulbright TM, Young RH. Metastatic carcinoma to the testis: a clinicopathologic analysis of 26 nonincidental cases with emphasis on deceptive features. Am J Surg Pathol. 2008;32(11):1683-93.

3. Kaplan MA, Kucukoner M, Inal A, et al. Testicular mass: An initial sign of squamous cell carcinoma of the lung. World J Oncol. 2012;3(6):291293.

4. Khan K, Bagchi D. Squamous cell carcinoma arising in a testicular teratoma and presenting as sister Mary joseph nodule. J Surg Tech Case Rep. 2011;3(2):99-101

5. Berney DM, Lu YJ, Shamash J, Idrees M. Postchemotherapy changes in testicular germ cell tumours: biology and morphology. Histopathology. 2017;70(1):26-39.

6. Bryan R.I. et al. Squamous cell carcinoma arising in a chronic hydrocele. Histopathology. 1990;17(2):178-180.

7. Albers P, Albrecht W, Algaba F, et al. European Association of Urology guidelines on testicular cancer. Eur Urol. 2011;60(2):304-19. 